\title{
Micellar Effects on the Photopolymerization of Acrylamide Sensitized by 2,3-Butanedione
}

\author{
Kaoru Iwai, Atsuko Matoike, Kiyomi Nishimuta, \\ and Fukuo TAKEMURA \\ Department of Chemistry, Faculty of Science, \\ Nara Women's University, Nara 630, Japan
}

(Received October 1, 1988)

\begin{abstract}
The photopolymerization of acrylamide sensitized by 2,3-butanedione (biacetyl: BA) was investigated in the presence of three surfactants with different types of hydrophilic groups. The rates of polymerization $\left(R_{\mathrm{p}}\right)$ in micellar solutions increased markedly by a factor of 3 to 4 in comparison with those in aqueous solutions, but the molecular weights of the polymers obtained $\left(\bar{M}_{n}\right)$ varied only slightly. In enough KSCN to quench the phosphorescence of BA, three surfactants showed their characteristic effects on the polymerizations depending on the nature of the hydrophilic head-groups. These findings are discussed and explained in terms of effects due to the incorporation of excited BA into micelles and electrostatic interactions of the charged radical species with the micellar surface.
\end{abstract}

KEY WORDS Photopolymerization / Acrylamide / Surfactant / Micelle / Sensitizer / 2,3-Butanedione / Biacetyl / Triplet Excited State / Degradative Chain Transfer/

As is well known, almost all examples of photopolymerization of vinyl monomers are of free-radical character and can be treated by simple kinetic equations derived for initiation, propagation, and biomolecular termination, going on the so called steady state assumption in radical concentrations. Neglecting the possible presence of such processes as primary radical termination and chain transfer, the over-all rate of polymerization $\left(R_{\mathrm{p}}\right)$ and average degree of polymerization $(\overline{\mathrm{DP}})$ can be expressed as

$$
R_{\mathrm{p}}=k_{\mathrm{p}}\left(\frac{2 \times 2.3 \phi \varepsilon d I_{0}}{k_{\mathrm{t}}}\right)^{1 / 2}[\mathrm{~S}]^{1 / 2}[\mathrm{M}]
$$

and

$$
\overline{\mathrm{DP}}=\frac{k_{\mathrm{p}}[\mathrm{M}]}{\left(2 \times 2.3 \phi \varepsilon d I_{0} k_{\mathrm{t}}\right)^{1 / 2}[\mathrm{~S}]^{1 / 2}}=\frac{k_{\mathrm{p}}{ }^{2}[\mathrm{M}]^{2}}{k_{\mathrm{t}} R_{\mathrm{p}}}
$$

respectively, where $k_{\mathrm{p}}, k_{\mathrm{t}},[\mathrm{M}],[\mathrm{S}], \phi, \varepsilon, d$, and $I_{0}$ are the rate constants of propagation and termination, the concentration of monomer and photosensitizer, quantum yield, extinction coefficient, light-pass length, and light intensity, respectively. ${ }^{1}$ Then at constant $[\mathrm{M}]$ and temperature under irradiation of stationary light, $R_{\mathrm{p}}$ should be proportional to the square root of $[\mathrm{S}]$ and $\overline{\mathrm{DP}}$ inversely proportional to $R_{\mathrm{p}}$.

However, in the previous paper ${ }^{2}$ it was reported that photosensitizer 2,3-butanedione (biacetyl: BA) and its excited state acted as a weak degradative chain transfer agent for the polymerization of acrylamide (AA) in aqueous solutions, and that the addition of an inorganic salt such as $\mathrm{KSCN}$ and $\mathrm{NaN}_{3}$ to this polymerization system increased not only $R_{\mathrm{p}}$ but also $\overline{\mathrm{DP}}$ of the polymer obtained. With respect to $\mathrm{KSCN}$, these effects were observed up to a concentration of the salt of $1 \times 10^{-4}$ moll $1^{-1}$, above which $R_{\mathrm{p}}$ was almost constant and the phosphorescence of BA was quenched 
completely. These findings could be best interpreted in terms of the removal of the influence of degradative chain transfer mainly through the triplet excited $\mathrm{BA}\left({ }^{3} \mathrm{BA}^{*}\right)$ and the formation of new initiating species $(\mathrm{SCN})_{2} \cdot{ }^{-}$ instead of $\mathrm{CH}_{3} \mathrm{CO} \cdot$ by means of the quenching of ${ }^{3} \mathrm{BA}^{*}$ by $\mathrm{KSCN}$. Therefore, separating ${ }^{3} \mathrm{BA}^{*}$ from the reaction-field of growing polymer radicals will be able to exclude the interaction between them without further addition of any salt. If such direct interactions between ${ }^{3} \mathrm{BA}^{*}$ and growing polymer radicals are removed by means of the incorporation of ${ }^{3} \mathrm{BA}^{*}$ in micelles, the degradative chain transfer mentioned above will be reduced and then the same effects with those of salts on $R_{\mathrm{p}}$ and $\overline{D P}$ can be expected.

Many papers on photochemical reactions in organized assemblies have been published..$^{3-13}$ With regard to photopolymerization, ${ }^{14-19}$ for example, Fouassier et al. ${ }^{17}$ reported higher $R_{\mathrm{p}}$ of acrylic monomers in an aqueous solution of sodium lauryl sulfate, and Kuo and coworkers $^{18}$ performed the photoinitiated polymerization of styrene in oil/water microemulsions using dibenzyl ketone as the initiator. These works were concerned with the acceleration of the propagation reaction by solubilization of water-insoluble monomers and initiators into micelles. Thus, the field, where polymer radicals are propagating the chains, is not an aqueous bulk solution phase, but a hydrophobic organic micelle core phase, which is not the case in this work.

In this report, characteristic kinetic behavior of the photopolymerization of AA sensitized by BA was studied in the presence of three kinds of surfactants with different hydrophilic head-groups. Further, the effects of KSCN added to these micellar polymerization systems were also studied. Then, a schematic picture for the effects of these surfactants on the BA-sensitized photopolymerization of AA can be given on the basis of the reactionenvironment of ${ }^{3} \mathrm{BA}^{*}$ and the species derived from the interaction with $\mathrm{SCN}^{-}$.

\section{EXPERIMENTAL}

\section{Materials}

Acrylamide (AA), 2,3-butanedione (biacetyl: BA), and inorganic salt $\mathrm{KSCN}$ were treated in a similar manner as before. ${ }^{2}$ Three different types of surfactants were sodium dodecyl sulfate (SDS) as an anionic surfactant, cetyltrimethylammonium chloride (CTAC) as a cationic one, and polyoxyethylene(9-10)p-octylphenyl ether (Triton $\mathrm{X}-100$ ) as a nonionic one, which were used as received and above the critical micelle concentration $(\mathrm{cmc})$.

\section{Experimental Procedures}

A freshly prepared sample solution degassed by four freeze-pump-thaw cycles was irradiated by $500 \mathrm{~W}$ ultra-high pressure mercury lamp with a cutoff glass filter $(85 \%$ transmittance at $365 \mathrm{~nm})$ at $30 \mathrm{C}$. The polymers were isolated by precipitation with methanol. Since the conversion was found to be almost linear with time, $R_{\mathrm{p}}$ was calculated from the weight of the dried polymer obtained by irradiation for 10 minutes and the average molecular weight of the polymer $\left(\bar{M}_{n}\right)$ was measured by GPC calibrated by poly(ethylene oxide) in water.

\section{Measurements}

The absorption and emission spectra of samples were recorded with a Hitachi model 124 spectrophotometer and a Hitachi model 850 fluorescence spectrophotometer, respectively, and absorption spectra of transient species under irradiation were monitored by the same flash photolysis apparatus as described previously. ${ }^{20,21}$

\section{RESULTS AND DISCUSSION}

\section{Effects of Surfactants on the Photopolymeri- zation of $A A$ Sensitized by $B A$}

As shown in Figure 1, the presence of any one of the surfactants with different hydrophilic groups increased $R_{\mathrm{p}}$ markedly by a factor of 3 to 4 in comparison with those in 


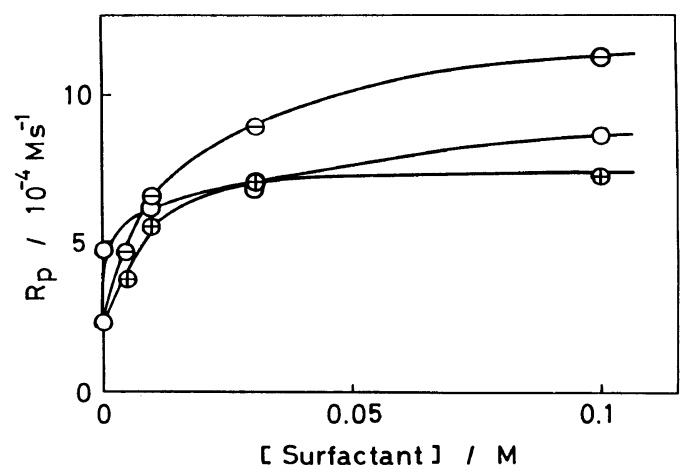

Figure 1. Dependence of the rate of photopolymerization on the concentration of the surfactant at $30^{\circ} \mathrm{C}: \ominus$, SDS; $\oplus$, CTAC; $\bigcirc$, Triton X-100; $[\mathrm{AA}]=1 \mathrm{moll}^{-1}$ and $[\mathrm{BA}]=0.1 \mathrm{moll}^{-1}$.

aqueous solutions. Especially, non-ionic surfactant Triton X-100 was very effective at low concentration. Moreover, $R_{\mathrm{p}}$ vs. [surfactant] curves showed pronounced saturation effects similar to those observed in enzyme-substrate systems. At a constant concentration of BA under irradiation of a fixed light intensity, ${ }^{3} \mathrm{BA}^{*}$ may be distributed in equilibrium between a micellar phase and an aqueous bulk phase. Since growing polymer radicals have been found to undergo degradative chain transfer with ${ }^{3} \mathrm{BA}^{*}$ in an aqueous bulk phase, ${ }^{2}$ quite large enhancement of rates can be considered to arise from incorporation of ${ }^{3} \mathrm{BA}^{*}$ into the micelles. Hence, the incorporated ${ }^{3} \mathrm{BA}^{*}$ might not act as a degradative transfer agent but as a precursor of initiating radicals. From analogy to Michaelis-Menten kinetics on the assumption that $R_{\mathrm{p}}$ is roughly linear to the concentration of ${ }^{3} \mathrm{BA}^{*}$ in micelle phase $\left[\left({ }^{3} \mathrm{BA}^{*}\right)_{\mathrm{m}}\right]$, a relationship between $R_{\mathrm{p}}$ and the micelle concentrations is derived as follows.

$$
\begin{gathered}
{ }^{3} \mathrm{BA}^{*}+\mathrm{MC} \rightleftharpoons \\
K_{\mathrm{m}}=\frac{\left[{ }^{3} \mathrm{BA}^{*}\right][\mathrm{MC}]}{\left[\left({ }^{3} \mathrm{BA}^{*}\right)_{\mathrm{m}}\right]} \\
\frac{R_{\mathrm{p}}{ }^{\mathrm{o}}}{R_{\mathrm{p}}}=\frac{\left[{ }^{3} \mathrm{BA}^{*}\right]_{0}}{\left[\left({ }^{3} \mathrm{BA}^{*}\right)_{\mathrm{m}}\right]}=1+\frac{K_{\mathrm{m}}}{[\mathrm{MC}]}
\end{gathered}
$$

Here, $\mathrm{MC},\left[{ }^{3} \mathrm{BA}^{*}\right]_{0}$, and $K_{\mathrm{m}}$ are the micelle, the

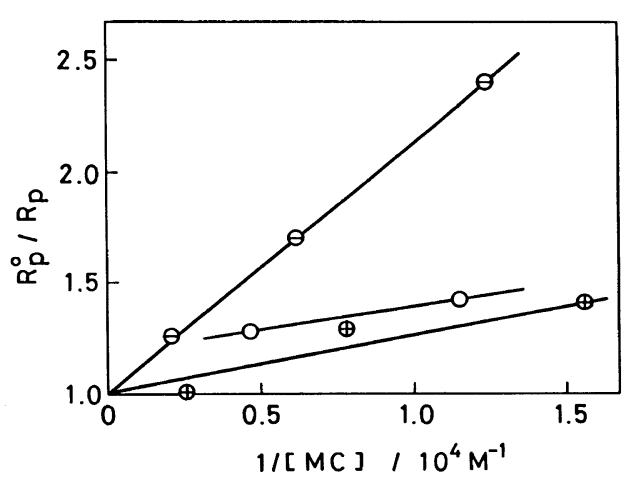

Figure 2. Plots of $R_{\mathrm{p}}{ }^{0} / R_{\mathrm{p}}$ against the reciplocal of the micellar concentration: $\ominus$, SDS; $\oplus$, CTAC; $\bigcirc$, Triton $\mathrm{X}-100 ;[\mathrm{AA}]=1 \mathrm{moll}^{-1}$ and $[\mathrm{BA}]=0.1 \mathrm{moll}^{-1}$.

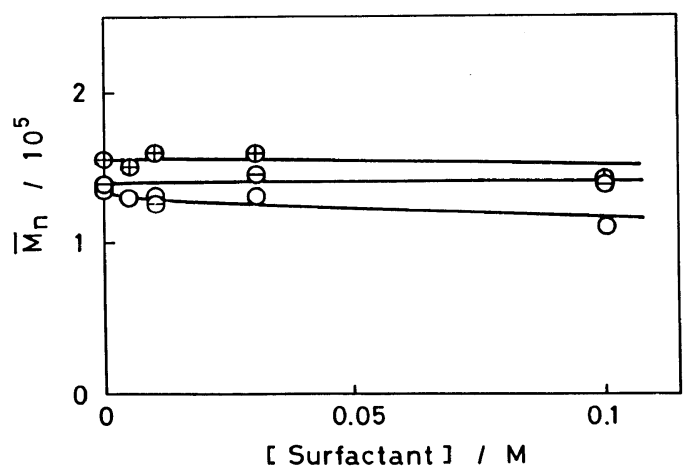

Figure 3. Dependence of the molecular weight of the polymer obtained on the concentration of the surfactant: $\ominus$, SDS; $\oplus$, CTAC; $\bigcirc$, Triton X-100; $[\mathrm{AA}]=1 \mathrm{moll}^{-1}$ and $[\mathrm{BA}]=0.1 \mathrm{moll}^{-1}$.

total concentration of ${ }^{3} \mathrm{BA}^{*}$, and an equilibrium constant corresponding to Michaelis constant, respectively. $R_{\mathrm{p}}{ }^{\circ}$ is the saturated rate in the case of no degradative chain transfer and the micelle concentration is taken as the surfactant concentration divided by the aggregation number (62 for SDS, 78 for CTAC, and 140 for Triton). ${ }^{4}$ It can be seen from Figure 2 that eq 1 is applicable to SDS and CTAC, for which $K_{\mathrm{m}}$ are $1.1 \times 10^{-4} \mathrm{moll}^{-1}$ and $2.6 \times 10^{-5} \mathrm{moll}^{-1}$, respectively, but is not in Triton $\mathrm{X}-100$ because of the strong quenching of ${ }^{3} \mathrm{BA}^{*}$.

$\bar{M}_{n}$ of the polymers obtained in the presence of any surfactant varied only slightly depending on the nature of the hydrophilic groups 


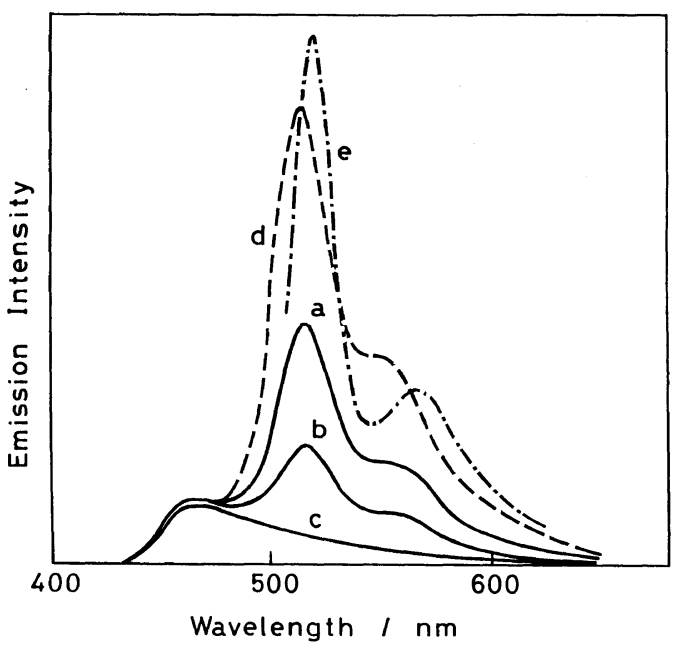

Figure 4. Emission spectra of BA in the presence of surfactants, in water, and in $n$-hexane: a, SDS $(0.1$ moll $\left.1^{-1}\right)$; b, CTAC $\left(0.1 \mathrm{moll}^{-1}\right)$; c, Triton X-100 (0.001 moll $\left.1^{-1}\right) ; \mathrm{d}$, in water; e, in $n$-hexane (at reduced scale $1 / 2) ;[\mathrm{BA}]=0.1 \mathrm{moll}^{-1}$.

and concentration (Figure 3). This will be discussed in following section.

Emission Spectra of $B A$ in the Presence of Surfactants

It is widely known that BA is soluble both in water and organic solvents and has a unique property of exhibiting phosphorescence as well as fluorescence in a fluid solution at room temperature. Therefore, in order to study the interactions between ${ }^{3} \mathrm{BA}^{*}$ and surfactants, the emission spectra of $\mathrm{BA}$ in aqueous micellar solutions were recorded along with those in water and in $n$-hexane as shown in Figure 4. In spite of no change of fluorescence intensity at $460 \mathrm{~nm}$, the phosphorescence at $513 \mathrm{~nm}$ was quenched moderately with the anionic surfactant (SDS), considerably with the cationic one (CTAC), and completely with the nonionic one (Triton X-100). Stern-Volmer constants $\left(K_{\mathrm{SV}}\right)$ for quenching ${ }^{3} \mathrm{BA}^{*}$ by these surfactants are listed in Table I cited later. It is noticeable that $K_{\mathrm{Sv}}$ for Triton X-100 is extremely large compared with those for SDS and CTAC. Since the maximum wave-lengths

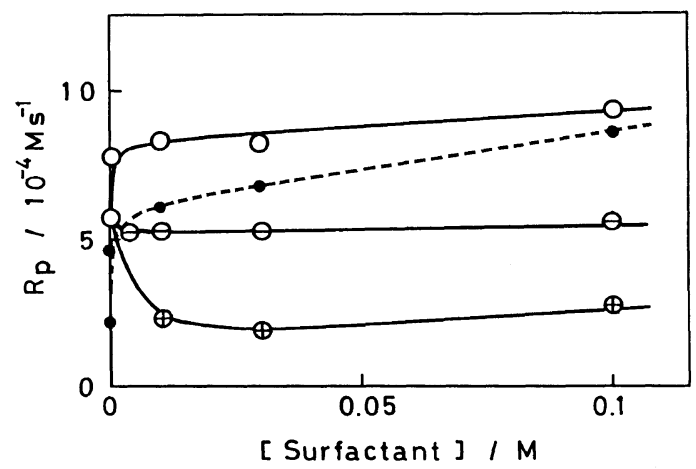

Figure 5. Dependence of the rate of photopolymerization on the concentration of the surfactant in the coexistence of KSCN: $\ominus$, SDS; $\oplus$, CTAC; $\bigcirc$, Triton X100 ; Triton $\mathrm{X}-100$ in the absence of $\mathrm{KSCN}$; $[\mathrm{AA}]=1$ moll ${ }^{-1},[\mathrm{BA}]=0.1 \mathrm{moll}^{-1}$, and $[\mathrm{KSCN}]=0.01 \mathrm{moll}^{-1}$ except for CTAC, for which $[\mathrm{KSCN}]=2 \times 10^{-4} \mathrm{moll}^{-1}$.

of the phosphorescence spectra of BA in these micellar solutions are similar to that in water rather than that in $n$-hexane, it seems that $\mathrm{BA}$ is located on the surface region of a micelle.

\section{Roles of Surfactants in the Photopolymerization Sensitized by $B A$}

In an aqueous micellar solution, BA is incorporated into a micelle and can be excited there on irradiation to yield radicals by either self-decomposition or hydrogen abstraction from surfactants. Thereafter, these radicals could initiate the polymerization of AA at the micellar surface and the resulting polymer radicals are present in the aqueous bulk solution and undergo propagation reaction with AA. Thus, the degradative chain transfer of the growing polymer radical with ${ }^{3} \mathrm{BA}^{*}$ is suppressed by virtue of the separation between them. Therefore, incorporation of ${ }^{3} \mathrm{BA}^{*}$ into micelles can be taken to be responsible for the increases in both $R_{\mathrm{p}}$ and $\bar{M}_{n}$.

Although BA-phosphorescence was quenched completely in a Triton X-100 micellar solution, photopolymerization was accelerated even at low micellar concentration. This suggests that the quenching is not due to a simple loss of the excitation energy by transfer reaction, but to the induced self-decomposition 
or hydrogen abstraction from surfactants of ${ }^{3} \mathrm{BA}^{*}$ leading to an increase in the concentration of initiating radicals. Consequently, this increases the growing polymer radicals and then decreases $\bar{M}_{n}$ because of faster bimolecular termination.

Accordingly, these two effects in micellar solutions, that is, separation of ${ }^{3} \mathrm{BA}^{*}$ from growing polymer radicals and facility of producing radicals from ${ }^{3} \mathrm{BA}^{*}$, are cooperative on $R_{\mathrm{p}}$, but cancel each other out on $\bar{M}_{n}$. So, despite the large increase in $R_{\mathrm{p}}, \bar{M}_{n}$ may be virtually invariant with increasing concentration of the surfactants as shown in Figure 3.

\section{Effects of Surfactants on the Photopolymeri- zation Sensitized by BA in the Presence of KSCN}

Although ${ }^{3} \mathrm{BA}^{*}$ was shown to play a role as a degradative chain transfer agent and consequently to retard the photopolymerization of AA in aqueous solution, either the addition of inorganic salt such as $\mathrm{KSCN}$ or the presence of surfactant was found to exclude the retarding effect of ${ }^{3} \mathrm{BA}^{*}$ and then to accelerate the polymerization. Thereupon, it may be worth while to estimate the effects on the polymerization of adding KSCN micellar solutions containing any one of three different hydrophilic surfactants.

Plots of $R_{\mathrm{p}}$ against the concentration of surfactants in the presence of enough amounts of KSCN to quench phosphorescence of ${ }^{3} \mathrm{BA}^{*}$ are shown in Figure 5. At a surfactant-free region, $R_{\mathrm{p}}$ was considerably high on account of the presence of KSCN. In the presence of anionic surfactant SDS, $R_{\mathrm{p}}$ was held almost constant independent of the concentration of the surfactant, but in the case of non-ionic surfactant Triton X-100, $R_{\mathrm{p}}$ increased significantly even at a very low concentration. Compared with the case in the absence of $\mathrm{KSCN}$, which is shown by the broken line in the figure, the effects of $\mathrm{KSCN}$ on $R_{\mathrm{p}}$ overlapped with those of the surfactant in the low concentrations of the surfactant, but the effects were relatively small in the high concentrations. On the other hand, the cationic micelle CTAC considerably depresses $R_{\mathrm{p}}$ even at moderately low concentrations. This can be explained by the attraction of the negative anion radicals $(\mathrm{SCN})_{2} \cdot-$ derived from the electron transfer between ${ }^{3} \mathrm{BA}^{*}$ and $\mathrm{SCN}^{-}$on the positively charged micellar surfaces, leading to decrease in the initiating radicals due to their faster recombination.

In a similar manner, the dependence of $\bar{M}_{n}$ on the concentration of surfactant is shown in Figure 6 with reference to that for Triton X100 in the absence of KSCN. $\bar{M}_{n}$ of the polymers obtained in any micellar solution containing KSCN were larger than those of the polymers in the same micellar solutions without $\mathrm{KSCN}$. This suggests that the growing polymer radicals did not undergo degradative chain transfer with ${ }^{3} \mathrm{BA}^{*}$ in an aqueous bulk phase probably because of deactivation by KSCN.

Although $\bar{M}_{n}$ slightly changed with increasing concentration of SDS or Triton X-100, it increased appreciably at $0.01 \mathrm{moll}^{-1}$ for CTAC, being held nearly constant over the high range of the concentration. In the case of CTAC, the increase in $\bar{M}_{n}$ probably corres-

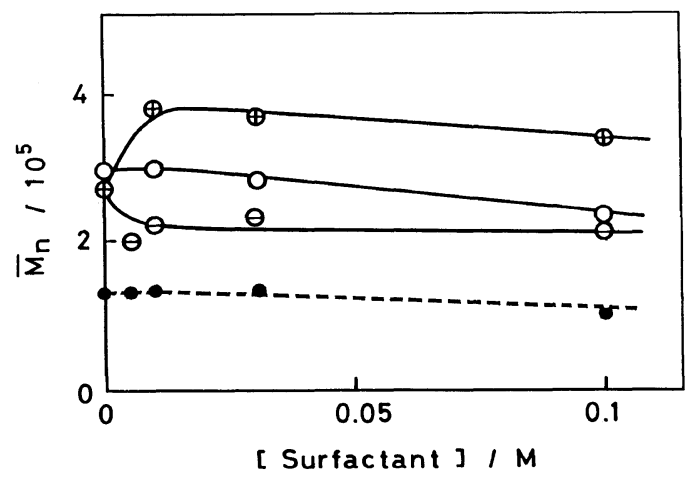

Figure 6. Dependence of the molecular weight of the polymer obtained on the concentration of the surfactant in the coexistence of $\mathrm{KSCN}: \ominus, \mathrm{SDS} ; \oplus, \mathrm{CTAC}$; $\bigcirc$, Triton X-100; $[\mathrm{AA}]=1 \mathrm{moll}^{-1},[\mathrm{BA}]=0.1 \mathrm{moll}^{-1}$, and $[\mathrm{KSCN}]=0.01$ moll ${ }^{-1}$ except for CTAC, for which $[\mathrm{KSCN}]=2 \times 10^{-4}$ $\mathrm{moll}^{-1}$. 
ponds to the decrease of $R_{\mathrm{p}}$ according to the normal kinetics of radical polymerization without degradative chain transfer.

\section{Profile of the Photopolymerization of $A A$} Sensitized by BA in the Coexistence of KSCN and Surfactants

As mentioned above, the photopolymerization of AA sensitized by $\mathrm{BA}$ in aqueous solution is depressed by the degradative chain transfer with ${ }^{3} \mathrm{BA}^{*}$, but in the presence of either an inorganic salt such as KSCN or a surfactant, the depression of $R_{\mathrm{p}}$ does not take place owing to either the quenching of ${ }^{3} \mathrm{BA}^{*}$ by salt or incorporation into micelles of ${ }^{3} \mathrm{BA}^{*}$. Therefore, the contributions of $\mathrm{KSCN}$ and surfactants to the changes of $R_{\mathrm{p}}$ and $\bar{M}_{n}$ can be compared with each other as follows. The values of $K_{\mathrm{Sv}}$ for the surfactants and KSCN are listed in Table I along with the variation in

Table I. Stern-Volmer constants of surfactants and KSCN for phosphorescence of BA and behavior of photopolymerization in $\mathrm{BA}-\mathrm{AA}-\mathrm{KSCN}$ Surfactant system ${ }^{a}$

\begin{tabular}{lccc}
\hline & SDS & CTAC & Triton \\
\hline$K_{\text {SV }}($ surfactant $) / \mathrm{M}^{-1}$ & 6 & 52 & $6 \times 10^{4}$ \\
$K_{\text {Sv }}(\mathrm{KSCN}) / \mathrm{M}^{-1}$ & $3 \times 10^{5}$ & $3 \times 10^{5}$ & $3 \times 10^{5}$ \\
Reference system & $\mathrm{BA}-\mathrm{AA}-$ & $\mathrm{BA}-\mathrm{AA}-$ & $\mathrm{BA}-\mathrm{AA}-$ \\
& $\mathrm{KSCN}$ & $\mathrm{KSCN}$ & Triton \\
$R_{\mathrm{p}}{ }^{\mathrm{b}}$ & $\rightarrow$ & $\downarrow$ & $\uparrow$ \\
$M_{n .}{ }^{\mathrm{b}}$ & $\rightarrow$ & $\uparrow$ & $\uparrow$ \\
\hline
\end{tabular}

a $[\mathrm{BA}]=0.1 \mathrm{moll}^{-1},[\mathrm{AA}]=1 \mathrm{moll}^{-1}$, and $[\mathrm{KSCN}]=$ $0.01 \mathrm{moll}^{-1}$.

b $\rightarrow$, unchanged; $\uparrow$, increasing; $\downarrow$, decreasing, compared with the reference system.
$R_{\mathrm{p}}$ and $\bar{M}_{n}$ with reference to the system cited. Since $K_{\mathrm{Sv}}$ for SDS and CTAC are very low compared with those for Triton X-100 and $\mathrm{KSCN}$, the effect of $\mathrm{KSCN}$ on the quenching of ${ }^{3} \mathrm{BA}^{*}$ is anticipated to be overwhelmingly greater than that of SDS or CTAC in a micellar solution containing $0.01 \mathrm{moll}^{-1}$ KSCN. Therefore, ${ }^{3} \mathrm{BA}^{*}$ both in an aqueous bulk phase and in a micelle phase can be quenched by $\mathrm{SCN}^{-}$generating transient anion radicals $(\mathrm{SCN})_{2} \cdot{ }^{-}$which are identified by the flash photolysis technique. ${ }^{20}$ In this connection, the ratios of the concentration of ${ }^{3} \mathrm{BA}^{*}$ in an aqueous bulk phase to that in a micelle phase for SDS and CTAC are estimated from the above-mentioned $K_{\mathrm{m}}$ to be $41 / 59$ and $17 / 83$, respectively, when the concentrations of surfactants are each $0.01 \mathrm{moll}^{-1} . K_{\mathrm{Sv}}$ for Triton X-100 is almost comparable to that for KSCN and then their contributions to the enhancement of the polymerization are nearly the same in magnitude. Therefore, it can be said that the effects of KSCN on both $R_{\mathrm{p}}$ and $\bar{M}_{n}$ can be superposed upon those of Triton X100 in the micellar solution, in which the initiating radicals might be produced by induced self-decomposition of ${ }^{3} \mathrm{BA}^{*}$ and hydrogen abstraction from the surfactants by ${ }^{3} \mathrm{BA}^{*}$.

The profiles of the polymerization sensitized by BA in aqueous micellar solutions containing KSCN are schematically shown in Figure 7. Thus, for SDS micellar solution anion radicals $(\mathrm{SCN})_{2} \cdot{ }^{-}$are present in an aqueous bulk phase to initiate the polymerization, but for CTAC solution, are concentrated on the pos-
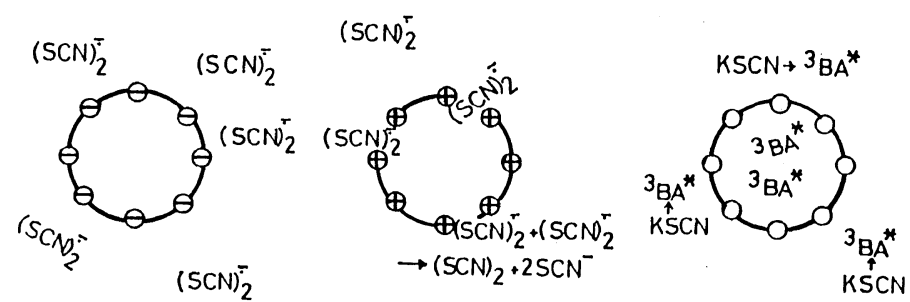

Figure 7. Schematic models for the behavior of initiating active species in the vicinity of micelleș: $\ominus$, SDS; $\oplus$, CTAC; $\bigcirc$, Triton X-100. 
itive micellar surfaces and disappear by mutual recombination. On the other hand, for non-ionic Triton $\mathrm{X}-100,{ }^{3} \mathrm{BA}^{*}$ can be remarkably quenched by both KSCN and the surfactant to yield $(\mathrm{SCN})_{2} \cdot{ }^{-}$and radicals resulting from ${ }^{3} \mathrm{BA}^{*}$. Then, these radicals can initiate the polymerization in parallel.

\section{CONCLUDING REMARKS}

In the presence of any one of three kinds of surfactants with different hydropholic headgroups, the photopolymerization of acrylamide (AA) sensitized by 2,3-butanedione (biacetyl: BA) was found to be enhanced by increasing the concentration of the surfactant up to the limiting rate, which corresponded to the rate without degradative chain transfer reaction between growing polymer radicals and ${ }^{3} \mathrm{BA}^{*}$. These effects of the surfactants can be ascribable to the protection of growing polymer radicals against degradative chain transfer and the promotion of production of initiating radicals from ${ }^{3} \mathrm{BA}^{*}$ by its incorporation into the micelle phase.

Furthermore, in sufficient $\mathrm{KSCN}$ to quench phosphorescence of BA, three surfactants showed characteristic behavior depending on the nature of hydrophilic head-groups. Although $R_{\mathrm{p}}$ was allowed to increase with the aid of KSCN in the surfactant-free aqueous solution, non-ionic surfactant Triton X-100 further increased $R_{\mathrm{p}}$ at a very low concentration and anionic SDS kept $R_{\mathrm{p}}$ almost constant over the range up to $0.1 \mathrm{moll}^{-1}$, but cationic CTAC considerably depressed the rates at moderately low concentrations concomitantly with increasing $\bar{M}_{n}$. These findings can be accounted for by the large $K_{\mathrm{SV}}$ of Triton X-100 and KSCN and by the electrostatic interactions of $(\mathrm{SCN})_{2} \cdot-$ derived from $\mathrm{SCN}^{-}$with charged micellar surface for both SDS and CTAC.

\section{REFERENCES}

1. F. W. Billmeyer, Jr., "Textbook of Polymer Science," John Wiley \& Sons, 3rd ed, New York, N. Y., 1984, p 56.

2. F. Takemura, K. Iwai, M. Ikesu, K. Itoh, and A. Matoike, Polym. J., 20, 565 (1988).

3. K. Kalyanasundaram, Chem. Soc. Rev., 7, 453 (1978).

4. T. Matsuo, Kagaku No Ryoiki, 32, 521 (1978).

5. J. K. Thomas, Chem. Rev., 80, 283 (1980).

6. J. H. Fendler, J. Chem. Educ, 60, 872 (1983).

7. J. Sunamoto, Kagaku Sosetsu, 40, 105 (1983).

8. D. G. Hall, J. Phys. Chem., 91, 4287 (1987).

9. F. M. Menger and C. E. Portnoy, J. Am. Chem. Soc., 89, 4698 (1967).

10. J. C. Scaiano, E. B. Abuin, and L. C. Stewart, J. Am. Chem. Soc., 104, 5673 (1982).

11. F. J. DeLuccia and L. J. C. Love, Anal. Chem., 56, 2811 (1984).

12. W. J. Dressick, B. L. Hauenstein, Jr., J. N. Demas, and B. A. DeGraff, Inorg. Chem., 23, 1107 (1984).

13. N. J. Turro, B. H. Baretz, and P. L. Kuo, Macromolecules, 17, 1321 (1984).

14. A. Merlin and J. P. Fouassier, Polymer, 21, 1363 (1980).

15. D. M. Moore and C. D. Burt, Photochem. Photobiol., 34, 431 (1981).

16. Y. S. Leong and F. Candau, J. Phys. Chem., 86, 2641 (1982)

17. J. P. Fouassier and D. Riviere, Polym. Photochem., 3, 29 (1983).

18. P. L. Kuo, N. J. Turro, C. Tseng, M. S. El-Aasser, and J. W. Vanderhoff, Macromolecules, 20, 1216 (1987).

19. C. K. Grätzel, M. Jirousek, and M. Grätzel, Langmuir, 2, 292 (1986).

20. F. Takemura and K. Sakaguti, Nippon Kagaku Kaishi, 1470 (1976).

21. K. Iwai, M. Uesugi, and F. Takemura, Polym. J., 17, 1005 (1985). 